\title{
STRATEGIES FOR ACADEMIC SUCCESS: AN EARLY INTERVENTION APPROACH FOR BUILDING METACOGNITIVE SKILLS IN FIRST-YEAR UNDERGRADUATE STUDENTS
}

\author{
Alexander S. Liepins and Carrie Hanson \\ Teaching and Learning Services, McGill University \\ alexander.liepins@mcgill.ca and carrie.hanson@mcgill.ca
}

\begin{abstract}
Strategies for Academic Success is a cocurricular workshop for first-year undergraduates on metacognitive skills and learning strategies that aims to support students' achievement of their learning goals. After multiple iterations, self-reported data has been collected, which allows us to examine and reflect on the learning strategies and habits that students have put into practice as a result of participating in the session, as well as whether the timing of session plays a role in determining the impacts of the content of study habits in students. In sum, we have found that certain strategies resonate more strongly with students based on whether they are entering university or have had at least one semester of university learning experience. Whereas there are broad applications for the strategies, knowing which strategies students gravitate toward relative to the student life cycle is useful for instructors and student success practitioners more generally.
\end{abstract}

Keywords: Metacognition, skills development, academic success, learning strategies, first-year undergraduates.

\section{INTRODUCTION}

University-level learning is complex and the transition from secondary to post-secondary education is not always easily effected by all students. There are many reasons and factors, but one postulate is a general lack of understanding regarding to how to learn best. Old or familiar study habits may or may not be effective, so a space to critically reflect on one's study habits, supplemented by exposure to a larger arsenal of learning strategies, can bolster the strategies that students can apply to foster their learning, their active engagement with course material, and their overall academic success. This is the rationale behind Strategies for Academic Success, a 60-minute workshop for first-year undergraduate students offered by McGill University's Teaching and Learning Services (TLS) student skills team.

The content of the workshop draws heavily from the work of Saundra Yancy McGuire [1] insofar as the argument that teaching students how to learn can have a positive impact on their learning and performance, which is the basis for the content delivered in the workshop. Other literature on effective learning strategies and self-regulated learning is also included in the workshop design. For example, the importance of metacognition for student development from Brown further provides guidance for strategies students can implement in their self-regulated learning practice [2]. Additionally, the importance of repeatedly revisiting material is emphasized through the Ebbinghaus forgetting curve [3].

McGuire encourages a learning skills intervention following the first assessment in coursework, particularly because the content is tied to a specific curricular context, but also because many first assessment results can be 'wake up calls' for some students, thereby making it slightly easier to reach students with the core elements of the power of learning strategies and metacognitive skills. In contrast, our work with Strategies for Academic Success examines the effectiveness of pre- versus post-assessment intervention for metacognitive skills in first-year undergraduates in the co-curricular context, i.e., outside the classroom. We argue that understanding the effect that the timing of the intervention has on undergraduate students allows instructors (and those supporting learners in other capacities) to know when to present and emphasize these strategies to students, as well as which strategies resonate the strongest at particular stages of the student life cycle. While the results in this case are self-reported and do not take into consideration the students' grades postintervention, they open opportunities for further research on the topic. In what follows we will summarize the workshop content and strategies, discuss our iterations, as well as adaptations of the session, and then outline and infer from survey results about the strategies students used as a result of their participation in the workshop.

\section{STRATEGIES FOR ACADEMIC SUCCESS}

In January 2019, TLS began offering Strategies for Academic Success with the aim of building metacognitive skills in first-year undergraduate students. The intent of 
this session is to support students with skills they can use to help them realize their academic goals and encourage deeper learning. Based on the framework from McGuire's Teaching Students How to Learn, and open to students from all Faculties at McGill, the goals of the Strategies for Academic Success workshop are for students to: (i) become aware of activities to facilitate their learning, (ii) become familiar with how to be strategic in their approach to studying, and (ii) use active learning strategies to empower their academic success.

Overall, the session takes a positive reinforcement approach about what students can do and aims to impart that changing behavior can change performance, i.e., if students want to succeed in their university courses, they need to be open to modifying their past or familiar study habits. The session is then framed as an opportunity to learn about theory- and research-backed strategies that are easy to implement and that will take any student from wherever they are now to where they want to be by enhancing their capacities to monitor and judge their learning as they go. Whether it is called metacognition or study skills, the main idea is to encourage deeper learning.

A total of 13 learning strategies are presented and summarized in the session. These strategies are supplemented by quick activities that aim to reinforce the idea that taking a strategic and goal-oriented approach to studying will facilitate their academic success. These 13 strategies are as follows:

1. Teaching material to others by either presenting to another person, real or imaginary;

2. Critical reading, which includes any combination of previewing the text, coming up with questions, then reading supplemented with recitation;

3. Preparing for class by previewing material and coming up with a question, attending class and being actively present, then reviewing notes and summarizing the lecture content;

4. Effective note-taking, for which taking notes by hand is encouraged to facilitate selectiveness about what material is recorded for future reference;

5. Summarization;

6. Paraphrasing in writing or by thinking out loud;

7. Flash cards, which can be created by hand or by using apps;

8. Concept mapping;

9. Optional homework;

10. Studying in pairs or groups;

11. Practice testing;

12. Creating test-worthy questions; and

13. Recitation.

A learning strategies self-assessment is also provided to students after the workshop, a resource included in
McGuire's Teach Students How to Learn, but which will not be further discussed here.

In addition to the presentation of the strategies and a brief elaboration of each, several active learning strategies are implemented to encourage student engagement with the content, such as the one-minute paper and think-pair-share strategies. Each of these activities focuses on a specific question, such as what strategies are you using already? What is the difference, if any, between studying and learning? And, at what level of Bloom's Taxonomy do you think you need to operate to be successful at McGill, as opposed to secondary school? As is suggested in McGuire's text, there is value in showing students the hierarchy of learning goals and having them engage with it through a question. We have also seen further benefits in using Bloom's as a foil for presenting the cycle of selfregulated learning because students who have a clear sense of what they are expected to know and do are better positioned to align study behaviors with their specific study goals.

The session wraps up with a summary of tips, like making the most of class time and asking questions, and then some institution specific resources. Finally, students are asked to create a quick action plan: What strategy are they going to try, why, and how and when will they implement it? Whereas we have not followed up to date on the specifics of their individual action plans, throughout the various iterations of this workshop we have always asked students which strategy they planned to put into practice. This data has been routinely collected for all iterations of Strategies for Academic Success and is summarized below.

\section{ITERATIONS AND ADAPTATIONS}

Since launching in January 2019, Strategies for Academic Success has been offered a total of seven times and at varied instances for first-year lifecycle. What distinguishes each offering is whether the student audience had already experienced one semester of university-level learning or not.

The pilot offerings occurred in January 2019 and were marketed toward first-year students who had just completed their first semester of university, but the session was open to all undergraduates, including newly admitted students for the Winter 2019 term. Two sessions were offered in partnership between TLS and McGill's Campus Life and Engagement offices. Since this was a pilot, information about students' Faculty and year of study was collected. A total of 60 students participated, representing 25 different departments from seven Faculties. This was, for all but three student participants, a second semester intervention.

The second iteration was offered in August 2019 as part of the Discover McGill orientation events as an early intervention. Three sessions were delivered as part of the academic expectations programming line-up. Complete 
attendance was not prioritized at these events, but based on a survey conducted during each workshop it is known that over 800 incoming undergraduate students attended this iteration of Strategies for Academic Success. Because the workshop had a key role in orientation, it was not offered again in Fall 2019.

Beginning in 2020, the third iteration of Strategies for Academic Success was delivered to 11 students across two sessions; again, this was a second semester intervention for most student participants. It is noteworthy that lower participation is expected in the Winter term, as it fits a larger trend for student skills development initiatives during the Winter months. The first session was in January and was open to all undergraduate students, but continued its original intent of being primarily marketed to first-year students. The second occurred in February and was offered in collaboration with the Residence Life team in McGill's residence halls, again for first-year undergraduates. Like the initial pilot in 2019, the attendees had all experienced at least one semester of University-level learning at McGill. Furthermore, in these two sessions the content had largely remained consistent from the initial pilot, but with minor changes to ordering and examples used to illustrate key topics. For example, Bloom's Taxonomy was presented before the concept of metacognition was introduced in order to provide the foundation for goalsetting, which underwrites the importance of setting intentional learning goals within the broader metacognitive skillset.

Beyond those three phases of offerings of Strategies for Academic Success, the workshop has undergone some adaptations since the January 2019 pilot initiative. For example, a 90-minute version of the workshop was offered to visiting international secondary school students in a special program run through McGill's Faculty of Education and was intended to be both an experience of university-level education, as well as university-level learning readiness. More recently, and as a result of the transition to remote learning due to the COVID-19 pandemic, Strategies for Academic Success was repurposed and offered as a webinar entitled Strategies for Remote Learning Success. Because those offerings did not explicitly target first-year students, those adaptations will not be considered further here. However, the adaptability of the content for adjusting to remote learning is noteworthy in that the only major revision was the addition of some items on virtual classroom communities, online communication, learning technology, in addition to the reinforcement that metacognition is pivotal for fostering responsibility and independence, especially for remote learning success.

\section{STRATEGY SURVEY RESULTS}

Each offering of Strategies for Academic Success asks students about which of the learning strategies they plan to implement in their study practice. The way in which this information has been collected varied across the offerings in terms of the timing, but the intent has been to determine what strategy or strategies resonate with attendees and, if possible, to draw inferences from that information. In what follows we will elaborate in a summary fashion the top strategies from the various offerings that students reported they planned to implement or that they implemented in their study practice. Then, we will discuss the results in terms of the timing of the workshop as an intervention to boost students' academic success.

Following the January 2019 pilot, a survey was sent to student participants from both sessions of the workshop to ask about the strategies that they were implementing. Although lasting habit cultivation and behavioral change takes time, we were primarily interested in what strategies resonated with students enough for them to attempt them in at least one of their study sessions. The survey was sent one week after the second of the two workshops concluded and, among other questions, asked students: Which of the following strategies have you used since attending the workshop? Students could select multiple strategies from the following list: Teaching material to others, critical reading, prepare-attend-review, effective note-taking (e.g., Cornell Notes), summarization/paraphrasing, concept mapping, optional homework, studying in pairs or groups, practice testing, creating test-worthy questions, recitation, the learning strategies inventory, none, or other.

Of the students who attended the January 2019 pilot, as seen in Fig. 1, 60\% $(n=36)$ completed the follow-up survey and self-reported that they attempted all the strategies at varying frequencies, but effective note-taking and concept mapping were implemented the least.

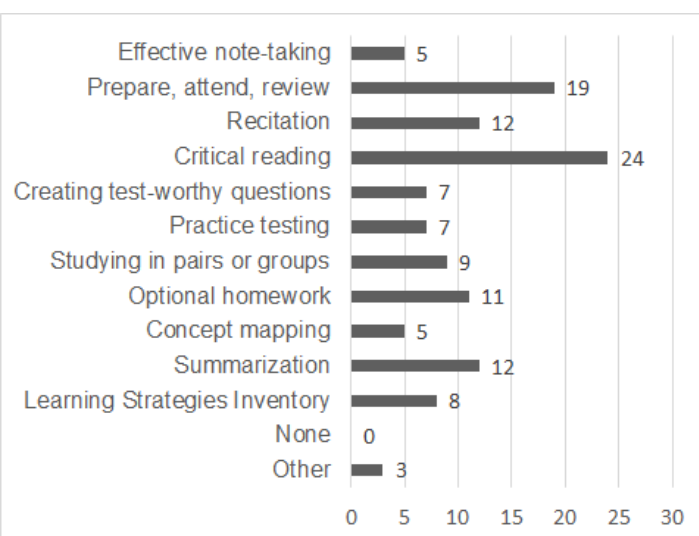

Figure 1: January 2019, second semester intervention, 36 responses

The three most popular strategies were critical reading $(n=24)$, prepare-attend-review $(n=19)$, followed by a tie between summarization and recitation $(n=12)$. No student who completed the follow up survey indicated that they had not tried any of the strategies. 
For the August 2019 sessions offered as part of orientation activities, the question about strategy implementation was embedded into the workshop, allowing students to respond via their cell phones immediately before the session concluded. This meant that 820 student participants in Strategies for Academic Success responded to the question: Which of the following strategies will you put into practice? Like the pilot followup survey, students could select multiple strategies, but for this version of the survey the learning strategies inventory was replaced with the flash cards strategy.

Of the students who completed the strategy survey during the orientation sessions, the three most selected strategies in order of popularity, as seen in Fig. 2, were effective note-taking $(\mathrm{n}=618)$, prepare-attend-review $(n=614)$, and optional homework $(n=610)$. The least selected strategy was concept mapping $(\mathrm{n}=197)$ followed by creating test-worthy questions $(n=414)$. Of the 820 survey responses, less than five indicated they would implement none of the strategies.

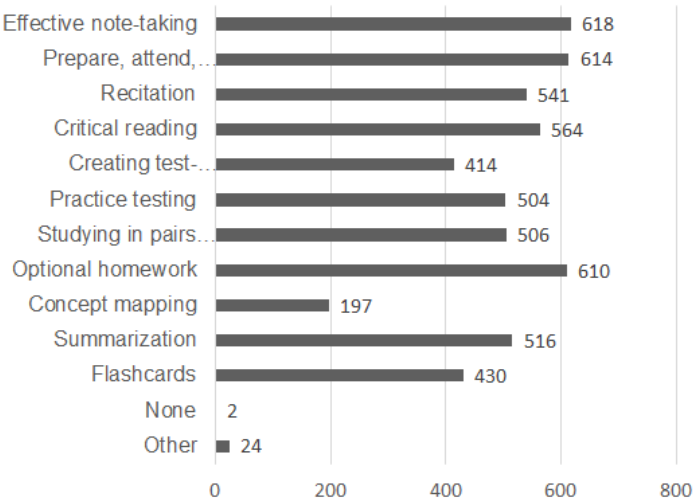

Figure 2: Orientation 2019, early intervention, 820 responses

After the Fall 2019 term (September through December), a follow up survey was sent to participants of the orientation sessions of Strategies for Academic Success. Figure 3 shows that about $11 \%(n=90)$ of students completed the survey, which posed the question: Which of the following strategies did you implement during the Fall 2019 semester? Students had the option again of selecting multiple strategies and the list of options was the same as for the August 2019 survey in the workshops.

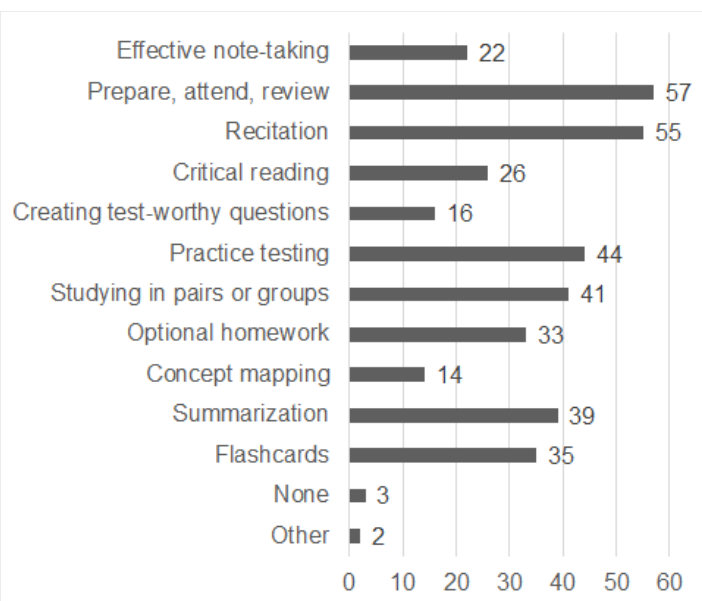

Figure 3: Orientation 2019, early intervention follow up, 90 responses

The top three strategies reported through the survey in order of popularity were prepare-attend-review $(n=57)$, recitation $(n=55)$, and practice testing $(n=44)$. And as with the results from August, the least selected strategy was concept mapping $(n=14)$, followed by creating test-worthy questions $(n=16)$. Less than five indicated they implemented none of the strategies listed in the survey.

The third iteration of Strategies for Academic Success was delivered to 11 students across two sessions. The method of using a survey during the workshop was continued. Students completed the strategy survey in response to the question: Which of the following strategies will you put into practice? Students could select multiple strategies, and the list of selections was consistent with the orientation survey. Due to the low sample size across the two sessions, it is difficult to explicitly determine the most and least popular strategies. However, prepare-attendreview, critical reading, teaching the material to others, and creating test-worthy questions had the most selections, whereas effective note-taking, concept mapping, optional homework, practice testing, and flash cards had the least. Less than 5 reported that they would not implement any of the strategies. It should also be added that there had been plans to offer more sessions during the Winter 2020 term, but this was disrupted due to changes in programming delivery as a result of COVID-19.

In what follows, we will discuss the results and draw some general inferences based on the data summarized above.

\section{DISCUSSION OF RESULTS}

The iterations of Strategies for Academic Success had the same facilitator and slide deck for each presentation. The strategies imparted were also consistent across sessions, with the exception of flash cards. Controlling for those variables, there are two notable differences. First, as stated above, the strategies that students indicated that they would implement was not consistent across sessions, 
particularly when considering the top three in terms of frequency in the strategy survey. Second, the audience varied from those with at least one semester of university coursework experience and those entering post-secondary education as first-year undergraduates. Therefore, do certain strategies resonate more with students entering university, as opposed to those with at least one semester of post-secondary education experience? And how could this impact the content and timing of the workshop as an intervention designed to support student's metacognitive skills development and academic success?

When comparing the second semester intervention pilot sessions to the early intervention orientation sessions survey results, a key difference is the top three strategies that students stated they would implement. Whereas students from the January 2019 pilot stated that one week following the workshop they implemented critical reading, prepare-attend-review, summarization, and recitation with the greatest frequency, students in the August 2019 orientation sessions selected prepare-attend-review, effective note taking, and optional homework with the greatest frequency.

From these results it is clear that some level of preparation for classes, followed by attending classes, and reviewing material afterward is not only an enticing strategy, but one that students actually commit to implementing in their study practice. This is confirmed in the follow up survey with the orientation participants, who selected prepare-attend-review as the strategy with the greatest frequency of application in their Fall semester study habits. It is possible that since attending classes is a fairly fundamental curricular exercise, students found this one to be the easiest to put into practice, since the level of behavioral change required is limited. More importantly, this strategy encourages students to scope course content before class to get a sense of context, main ideas, and potentially even generate some questions to bring to class. Once in the class, students are not encountering the material for the first time, which boosts their ability to critical engage with it. Following class, by reviewing their notes or what was discussed, even if only briefly, they consolidate the material and potentially identify further questions or areas of focus for their studying - all of this illustrates a student's own critical reflection on their learning before, during, and after class, thereby supporting their metacognitive skills abilities and development.

One other notable takeaway from these results surrounds the commitment to optional homework. Students in the orientation session stated they would try strategies that generally cover going to classes, taking notes, and completing homework - familiar activities for secondary school students. However, after they completed their Fall term at McGill, the practice of effective note-taking dropped in frequency, along with optional homework. Additionally, in the surveys for students who had already completed at least one semester, $31 \%(n=11)$ stated they planned to implement optional homework; far fewer than the orientation group of whom $74 \%(n=610)$ indicated that they would complete optional homework. The commitment to optional homework from the orientation group could be due to a misunderstanding about the level of work required for university learning relative to time management skills, which is a key part of post-secondary education rigor, but such a conclusion has not been validated in the context of Strategies for Academic Success.

Furthermore, the results for the January 2019 pilot survey can be generalized to the point that students from those two sessions actually attempted strategies related to reading, attending classes, and making course content meaningful for them - a powerful learning consolidation strategy. When compared with the January 2020 follow up survey with early intervention orientation participants, there are more similarities than with the orientation session results. In both situations, students had experienced at least one semester of university and indicated that the strategies they were using included making the most of class time and making the material meaningful for themselves through recitation. Additionally, practice testing appeared with a larger frequency, which might be attributed to the nature of the assessment activities or course material - for example, STEM students might be completing more problem sets as part of course-related activities than students from programs who might have more reading and writing assignments. A further breakdown of students by faculty would be necessary, however, to explore such a claim.

All in all, the comparison of the results above makes the timing of metacognitive skills development interventions a question worth pursuing because if certain strategies resonate more strongly with students at different stages of post-secondary education, then that provides grounds for emphasizing some over others, depending on the cohort and the timing. McGuire recommends that learning skills interventions occur following the first assessment, particularly within the context of specific courses - this helps with clarifying the relevance and application of learning strategies to the specific courses. Our approach, however, is co-curricular and has benefited from being able to attempt these interventions at different stages relative to the first-year student life cycle. The novelty of the orientation approach is that it provides an early intervention toward the cultivation of metacognitive skills in students before their participation in any formal university level instruction or assessment, which might explain why certain strategies resonated more with those students, as compared with students who already had one semester of university-level experience.

\section{FUTURE DIRECTIONS}

There are several future directions to take this work, in addition to the fact that the COVID-19 pandemic 
interrupted planned offerings of the workshop and as well our abilities to consider student data related to faculty and departmental affiliations - coding by those factors would allow further inferences founded on the data summarized above.

We also intend to collect information about how the strategies were implemented and whether or not, after having completed their first semester at McGill, they perceive their participation in the 'Strategies for Academic Success' workshop as a valuable experience. This information will be used to improve the session for future iterations, including timing and the strategies that are emphasized in the workshop, toward the goal of fostering students' ownership of their learning, both inside and outside the classroom.

Another next step could be to look at the marks received by students who participated in any of these sessions to examine the students' 'academic success' insofar as it relates to GPA. For the second semester intervention, marks could be compared from before and after the intervention. For early intervention students, the marks could be compared to the average for first semester students to see if there is any correlation between early intervention and early academic success. This future direction would be difficult for a larger inter-faculty program such as this, but it is perhaps more possible to measure the success of a Faculty-embedded academic success program. Even if only self-reported, it would add to the data sets existing around student satisfaction with their experience in the workshop and subsequent behavioral changes.

Finally, an ideal situation would be to follow these students through their academic journey at McGill and have them regularly self-report on the strategies they still implement. This would not only track the effectiveness and longevity of the strategies introduced, but also allow the students to again reflect on their learning and therefore continue the process of self-regulated learning.

\section{CONCLUSION}

In sum, we have demonstrated success in conveying proven learning strategies to students through the workshop, particularly in the form of an early intervention as part of orientation activities. It is significant that students have reported that these lessons have transferred to their study behaviors. Furthermore, it is clear that students' own understanding of university learning and which strategies are appropriate to achieve their academic goals varies based on where they are in the first-year life cycle. The timing of these interventions also suggests that content emphases should consider which strategies students expect to implement and confirms McGuire's view that the intervention's timing relative to assessment is critical. Overall, this work has broad application and in the current context of remote teaching we expect metacognitive skills to play a key role in students continued university success at all stages in the student life cycle.

\section{References}

[1] Stephanie McGuire and Saundra Yancy McGuire, Teach Students How to Learn: Strategies You Can Incorporate into Any Course to Improve Student Metacognition, Study Skills, and Motivation. Sterling, VA: Stylus Publishing, LLC, 2015, pp. 240.

[2] Peter. C Brown, Make It Stick: The Science of Successful Learning. Cambridge, MA: Harvard University Press, 2014, 313 pp.

[3] Jaap M.J. Murre and Joeri Dros, "Replication and analysis of Ebbinghaus' forgetting curve," PLOS ONE, vol. 10 no. 7, 2015. https://doi.org/10.1371/journal.pone.0120644 